\title{
AVULSÃO DO PLEXO BRAQUIAL EM CÃES -1. ASPECTOS CLÍNICOS E NEUROLÓGICOS ${ }^{1}$
}

\author{
BRACHIAL PLEXUS AVULSION IN DOGS -1. \\ CLINICAL AND NEUROLOGICAL ASPECTS
}

\author{
Mônica Vicky Bahr Árias² Angelo João Stopiglia ${ }^{3}$
}

\section{RESUMO}

A avulsão do plexo braquial é afecção de ordem traumática relativamente comum, ocasionando paralisia grave do membro torácico. É freqüentemente confundida com paralisia do nervo radial, havendo controvérsias sobre o tratamento. $O$ objetivo deste trabalho foi: avaliar clinica e neurologicamente cães com avulsão do plexo braquial, demonstrando os aspectos significativos para o diagnóstico desta afecção. Observou-se predominância de cães sem raça definida, fêmea, com menos de três anos de idade, sendo o atropelamento a etiologia principal. As alterações clinicam/neurológicas mais freqüentes foram: paralisia flácida, ausência do reflexo dopanículo, ausência dos reflexos tricipital, bicipital e extensor do carpo radial, atrofia dos músculos tríceps, bíceps, supra-espinhal, infra-espinhal $e$ extensores do carpo, anestesia cutânea abaixo do nível do cotovelo e abrasão/ulceração em face dorsal da mão. A associação destes resultados com os aspectos da histologia e da eletroneuroestimulação (relatados na parte 2 e 3 deste trabalho, respectivamente) sugeriu envolvimento quase que total das raizes do plexo braquial em todos os casos.

Palavras-chave: avulsão do plexo braquial, cães, sistema nervoso periférico.

\section{SUMMARY}

Brachial plexus avulsion is a relatively common affection, causing serious paralysis of the thoracic limb. It is often misdiagnosed as radial paralysis and there are controversies about the treatment. The main purposes of this work were: to evaluate

\begin{abstract}
clinically and neurologically dogs with brachial plexus avulsion and to demonstrate the relevant aspects in the diagnosis of this affection. Predominantly mixed breed dogs, females under three years of age were observed, and the brachial plexus avulsion was mainly a result of road accidents. The more frequent clinical and neurological signs were: flacid paralysis, loss of the panniculus, triceps, biceps and extensor carpi radial muscle reflexes, atrophy of the muscles triceps, biceps, extensor carpi radial, supraspinatus and infraespinatus; superficial skin sensorial loss distal to the elbow; dorsal carpal skin abrasions and ulceration. The association of these results with histologic and electroneurostimulation exams (described in part 2 and 3 of this work respectively) suggested in all cases an almost total involvement of brachial plexus roots.
\end{abstract}

Key words: brachial plexus avulsion, dogs, peripheral nervous system.

\section{INTRODUÇÃO}

As desordens do plexo braquial em cães são observadas ocasionalmente na prática clínica, sendo constituídas por avulsão das raízes nervosas (GRIFFITHS, 1974; GRIFFITHS et al., 1974), neoplasias (WHEELLER $\boldsymbol{e}$ t al., 1986; SHORES, 1993), lesão de tronco nervoso específico (DE LAHUNTA, 1983), e neuropatia do plexo braquial (BRAUND, 1994).

\footnotetext{
${ }^{1}$ Parte da tese apresentada pelo primeiro autor como requisito parcial para obtenção do título de Mestre junto ao Departamento de Cirurgia, Faculdade de Medicina Veterinária e Zootecnia (FMVZ), Universidade de São Paulo (USP). Apoio bolsa de estudos da FAPESP.

${ }^{2}$ Médico Veterinário, Professor Assistente, Departamento de Anatomia, Centro de Ciências Biológicas, Universidade Estadual de Londrina, Caixa Postal 6001, 86051-970, Londrina - PR. Autor para corrspondência.

${ }^{3}$ Médico Veterinário, Professor Associado, Departamento de Cirurgia, FMVZ, USP.
} 
A avulsão das raízes constitui-se na desordem do plexo braquial de ordem traumática mais comum em cães, estando associada normalmente a atropelamentos. A lesão ocorre por tração do membro, o que gera forças longitudinais ao longo dos nervos. Assim, raízes nervosas que formam o plexo braquial, seus ramos ventrais e o próprio plexo braquial podem ser lesionados (SUMMERS et al., 1995). Para HOFFMANN $\boldsymbol{e}$ t al. (1990), a avulsão das raízes da medula espinhal ocorre na área de transição entre o sistema nervoso central e periférico.

Devido ao início súbito dos sinais clínicos e associação com traumatismos, o diagnóstico é simples. A maior dificuldade está em se determinar à extensão da lesão, isto é, quais são as raízes e nervos particularmente envolvidos. Esta determinação é importante para formular um prognóstico para recuperação da função, uma vez que na maioria dos casos a falta de um exame mais apurado tem levado à amputação do membro afetado (BAILEY, 1984), ou ao diagnóstico errôneo de paralisia do nervo radial (SUMMERS $\boldsymbol{e t}$ al., 1995).

A avulsão pode ser incompleta, pois ocorre em alguns casos a resistência de certos elementos teciduais (GRIFFITHS, 1974). Certas radículas podem ser destacadas, enquanto que outras pertencendo ao mesmo segmento medular podem permanecer íntegras (SUMMERS et al., 1995). As raízes são mais suscetíveis do que os nervos espinhais. Acredita-se que pelo fato de possuírem quantidade menor de perineuro apresenta menor resistência ao estiramento (BRAUND, 1987, SHORES, 1993).

O diagnóstico desta afecção baseia-se na anamnese, exames clínico-neurológico e complementares (BRAUND, 1986), sendo comumente relatado perda da função do membro imediatamente após o trauma. O sinal clínico mais evidente é a incapacidade de estender o cotovelo ou sustentar o peso sobre o membro afetado e o cão freqüentemente arrasta o dorso da extremidade do membro no solo. Observa-se então síndrome neuropática ou de neurônio motor inferior caracterizada por tônus muscular e reflexos segmentares ausentes ou diminuídos, e atrofia muscular neurogênica (SHORES, 1993).

O envolvimento da raiz do segmento medular torácico $1\left(\mathrm{~T}_{1}\right)$ que contém fibras préganglionares simpáticas freqüentemente resulta em síndrome de Horner parcial, com miose ipsilateral (HOERLEIN, 1978; BRAUND, 1986; CHRISMAN, 1989). Já uma lesão entre o segmento medular cervical $8\left(\mathrm{C}_{8}\right)$ e $\mathrm{T}_{1}$ pode produzir perda do reflexo do panículo ipsilateral (WHEELER et al., 1986).
Avulsão das raízes caudais entre $\mathrm{C}_{8}$ e $\mathrm{T}_{2}$ permite que o paciente "carregue" o membro com o cotovelo e ombro flexionados, visto haver função parcial dos nervos músculo cutâneo, axilar e supraescapular (SHORES, 1993). Para SIMPSON et al. (1985) e STEIMBERG (1988), praticamente todos os animais com avulsão das raízes do plexo braquial terão comprometimento dos nervos radial, mediano e ulnar, sendo que os nervos axilares e músculo cutâneo são freqüentemente, mas nem sempre afetados.

Os dermátomos são utilizado para localizar áreas de analgesia no membro afetado (SHORES, 1993), existindo alguns padrões básicos de anestesia cutânea nas lesões ao plexo braquial (KNECHT \& ST. CLAIR, 1969; GRIFFITHS et al., 1974; BAILEY, 1984).

A importância do diagnóstico correto é clara, já que, enquanto a paralisia do nervo radial pode ser tratada por artrodese do carpo, transposição muscular ou mesmo neurorrafia (STOPIGLIA \& ERHART, 1987), as avulsões tem prognóstico reservado, visto tais técnicas não serem eficientes para se obter o retomo, mesmo que parcial, das funções motora e sensorial. Estes aspectos incentivaram CARLSTEDT et al. (1993) a pesquisarem em primatas técnicas cirúrgicas de reimplante das raízes avulsionadas.

Indica-se a realização da amputação se houver analgesia distai ao cotovelo, automutilação, e quadro inalterado por três semanas. Caso haja melhora do quadro, o paciente é reavaliado a cada três semanas, prosseguindo-se com o tratamento conservador. Este pode ser mantido indefinidamente se o membro não estiver sujeito a traumatismos, e se a aparência do membro for aceitável para o proprietário (SHORES, 1993). Segundo WHEELER et al., (1986) e STEIMBERG (1988) alguns cães manteriam locomoção razoável com o membro afetado, devendo ser observados por vários meses antes de se decidir pela amputação.

O objetivo deste trabalho foi avaliar clínica e neurologicamente cães com avulsão do plexo braquial, demonstrando as características significativas para o diagnóstico dessa afecção.

\section{MATERIAIS E MÉTODOS}

Foram utilizados 12 cães de ambos os sexos, sendo oito fêmeas e quatro machos, com idade variando entre 6 meses e 11 anos, trazidos ao Hospital Veterinário da Faculdade de Medicina Veterinária e Zootecnia da Universidade de São Paulo por apresentarem quadro clínico compatível com avulsão do plexo braquial. 
Os 12 cães foram submetidos a exame clínico rotineiro e exame neurológico completo. O exame neurológico seguiu técnica descrita por BRAUND (1994), com atenção particular aos seguintes aspectos: distribuição da atrofia muscular, presença de síndrome de Horner, presença do reflexo do panículo e sensibilidade cutânea.

Os cães submetidos ao tratamento pelo método conservador (utilização de um dispositivo para sustentar o carpo, colar elizabetano e fisioterapia) eram reavaliados periodicamente, observando-se os progressos quanto à função motora e sensorial. Conforme a evolução do quadro optouse por panartrodese do carpo, seguindo-se técnica descrita por GRUMADAS (1987) ou manutenção do tratamento conservador mencionado anteriormente. Os animais submetidos a panartrodese do carpo foram acompanhados durante o período pósoperatório, avaliando-se periodicamente a capacidade de flexionar o cotovelo, a ocorrência de automutilação e a deambulação. Indicou-se a amputação alta (com retirada da escápula) do membro afetado, seguindo-se técnica cirúrgica descrita em ARCHIBALD (1974), quando havia úlceras profundas e automutilação grave.

\section{RESULTADOS}

Observou-se predominância de cães sem raça definida $(91,7 \%)$, fêmeas $(66,7 \%)$, com menos de 3 anos de idade (75\%), sendo o atropelamento a etiologia principal (75\%). A duração dos sinais clínicos na época da primeira consulta variou de três dias até dois anos e meio, sendo a maior parte dos pacientes $(66,7 \%)$ trazidos com um quadro clínico de até 30 dias de duração.

Os dados referentes a frequiência dos sinais clínicos e neurológicos encontram-se na Tabela 1 .

Constatou-se que todos os cães apresentavam paralisia flácida do membro afetado, sendo que quatro deles mantinham a capacidade de flexionar o cotovelo e dois flexionavam-no parcialmente. Em quatro destes seis cães, a flexão do cotovelo não preveniu a abrasão da face dorsal da mão. No geral $75 \%$ dos pacientes apresentaram esta mesma ulceração.

Todos os cães apresentaram ausência do reflexo do panículo ipsilateral ao membro afetado, havendo entretanto contração do músculo cutâneo do tronco contralateral. Observou-se também ausência dos reflexos tricipital, bicipital e extensor do carpo radial em todos os cães, enquanto que o reflexo interdigital estava parcialmente presente em cinco cães $(41,7 \%)$.

Observou-se atrofia do músculo tríceps em $75 \%$ dos cães, enquanto que os músculos extensores do carpo estavam atrofiados em $66,7 \%$ dos pacientes. Atrofia neurogênica marcante de músculos não inervados pelo nervo radial (supraespinhal, infraespinhal e bíceps) foi constatada em $66,7 \%$ dos animais. Anquilose em cotovelo e carpo e síndrome de Horner, representada por miose, foram constatados em $25 \%$ dos casos.

A avaliação subjetiva da sensibilidade cutânea demonstrou aspectos variados (Figura 1). Observou-se padrão de sensibilidade cutânea compatível com avulsão parcial (anestesia abaixo do nível do cotovelo, com preservação de pequena área normal em face medial de antebraço) em três cães. Padrão de sensibilidade cutânea compatível com avulsão total (anestesia abaixo do nível do cotovelo) foi verificada em cinco cães. Três cães apresentaram anestesia abaixo do nível do cotovelo e na face crânio-lateral de úmero, também compatível com avulsão total. Já um cão apresentava normo à hiperestesia acima da articulação do carpo, não sendo possível a avaliação de carpo e dígitos devido à grave automutilação desenvolvida.

Os dados referentes ao tratamento instituído em nove dos doze animais encontram-se na Tabela

Tabela 1 - Freqüência dos principais sinais clínicos e neurológicos em 12 cães portadores de avulsão do plexo branquial trazidos ao Hospital Veterinário da Faculdade de Medicina Veterinária e Zootecnia da USP.

\begin{tabular}{lcc}
\hline Sinal clínico/neurológico & Freqüência & $\%$ \\
\hline $\begin{array}{l}\text { Paralisia flácida / Ausência dos reflexos do panículo, } \\
\text { tricipital, bicipital e extensor carpo radial }\end{array}$ & 12 & 100,0 \\
$\begin{array}{l}\text { Úlceras ou abrasão em face dorsal de mão / Atrofia do } \\
\text { músculo tríceps }\end{array}$ & 9 & 75,0 \\
$\begin{array}{l}\text { Atrofia dos músculos supra e infraespinhal, bíceps e } \\
\text { extensores do carpo. }\end{array}$ & 8 & 66,7 \\
$\begin{array}{l}\text { Presença parcial do reflexo interdigital } \\
\text { Flexão do cotovelo }\end{array}$ & 5 & 41,7 \\
$\begin{array}{l}\text { Anquilose em cotovelo / Anquilose em carpo / Sídrome } \\
\text { de Horner }\end{array}$ & 3 & 33,3 \\
$\begin{array}{l}\text { Automutilação / Flexão parcial do cotovelo / Fratura em } \\
\text { outro membro }\end{array}$ & 2 & 16,7 \\
\begin{tabular}{l} 
Fratura em mesmo membro / Ulceração em cotovelo \\
\hline
\end{tabular} & 1 & 8,3 \\
\hline
\end{tabular}


2. Indicou-se ainda a amputação do membro em dois cães e artrodese do carpo em um.

Um dos pacientes submetidos a artrodese

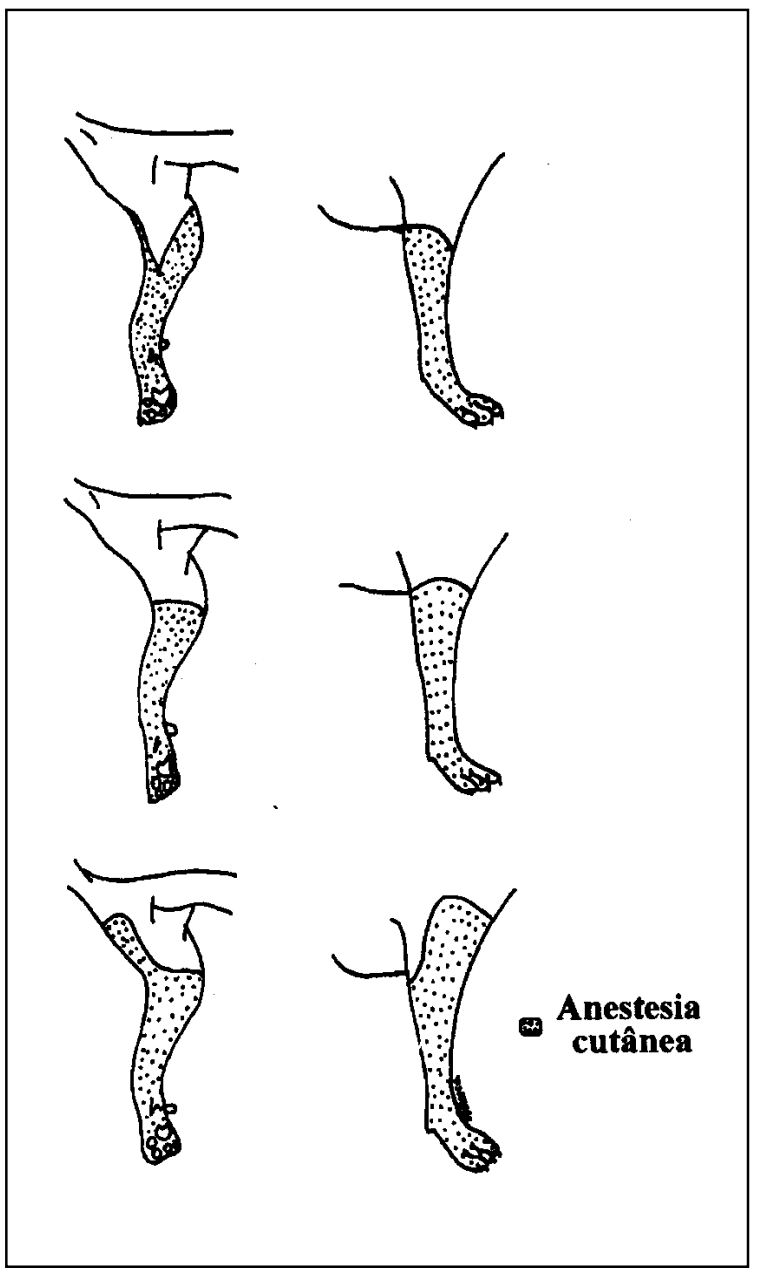

Figura 1 - Padrões de sensibilidade observados em cães com avulsão do plexo branquial.

Tabela 2 - Frequiência dos tratamentos realizados em nove cães portadores de avulsão do plexo branquial, trazidos ao Hospital Veterinário da Faculdade de Medicina Veterinária e Zootecnia da Universidade de São Paulo.

\begin{tabular}{lcc}
\hline Tratamento & Freqüência & $\%$ \\
\hline Amputação & 6 & 66,7 \\
Artrodese do carpo & 2 & 22,2 \\
Conservador & 1 & 11,1 \\
\hline Total & 0 & 100 \\
\hline
\end{tabular}

do carpo, após a cirurgia, deambulava com o cotovelo flexionado, raramente apoiando a extremidade do membro no solo, não apresentando abrasão em carpo. Periodicamente iniciava automutilação em dígitos sendo o proprietário orientado sobre o manejo adequado nestas ocasiões. Após cinco meses o quadro motor manteve-se inalterado, havendo progressão da sensibilidade cutânea do cotovelo para terço médio de antebraço. $\mathrm{O}$ segundo paciente submetido à artrodese foi avaliado durante três meses, sendo observado apoio mais freqüente do membro ao solo e posteriormente ulceração em face lateral da mão.

\section{DISCUSSÃO}

A observação de que a avulsão do plexo braquial acomete principalmente fêmeas jovens, sem raça definida, permite a sugestão de que medidas adequadas de manejo em relação ao acesso à rua, controle populacional e de animais durante o estro, podem diminuir o número de atropelamentos e a incidência desta doença.

Segundo SIMPSON et al. (1985), KNECHT \& RAFFE (1985), e SHORES (1993), a alteração neurológica mais óbvia é a perda da função motora do membro, paralisia flácida, incapacidade de estender o cotovelo, carpo e dígitos. Estes mesmos aspectos foram observados em todos os pacientes, havendo entretanto em seis cães (50\%) a capacidade de flexionar total ou parcialmente o cotovelo. Este resultado é semelhante ao obtido por VAN NES (1986), que observou flexão voluntária do cotovelo em 46,2\% dos casos, sendo isto conseqüente à função parcial do nervo músculo cutâneo.

Para KNECHT \& RAFFE (1985) e SHORES (1993), a perda da função do nervo ulnar, que fornece inervação sensorial à face lateral da mão, predispõe à ulceração acral quando o membro se desloca lateralmente. Esta lesão, observada na maioria dos casos, foi observada também em $66,7 \%$ dos animais que mantiveram capacidade de flexionar o cotovelo, demonstrando ser necessário perfeita integração entre funções motora e sensorial.

A porcentagem de animais com ausência do reflexo do panículo ipsilateral foi semelhante aos resultados obtidos por GRIFFITHS et al. (1974) e WHEELER et al. (1986) que a constataram em 94,4\% e $91,7 \%$ dos casos, fornecendo assim informações sobre a extensão da lesão na porção caudal do plexo braquial. O efetor deste reflexo, o músculo cutâneo do tronco, é inervado pelo nervo torácico lateral, que se 
origina dos segmentos medulares $\mathrm{C}_{8}$ a $\mathrm{T}_{1}$ ). Este resultado corrobora o fato do nervo ulnar estar lesado em todos os casos, pois sua origem situa-se entre os segmentos medulares $\mathrm{C}_{8}$ e $\mathrm{T}_{2}$.

A ausência dos reflexos tricipital, extensor do carpo radial e bicipital em todos os casos indicou freqüente acometimento do nervo radial e músculo cutâneo. Para BRAUND (1994), os reflexos tricipital e bicipital são difíceis de serem realizados, podendo estar ausentes em cães normais. Enquanto que o reflexo extensor do carpo radial é o mais seguro na avaliação da função do nervo radial, para avaliação do nervo músculo cutâneo deve ser observado a capacidade de flexionar ativamente o cotovelo.

A anquilose em cotovelo e carpo para KNECHT \& RAFFE (1985) resulta da contratura dos tendões flexores devido à falta de fisioterapia adequada, e conforme recomendado por estes autores e VAN NES (1986), a amputação do membro afetado foi o procedimento adotado neste trabalho.

Segundo BRAUND (1994), o envolvimento das raízes de $\mathrm{T}_{1}$ à $\mathrm{T}_{3}$ que contém fibras pré-ganglionares simpáticas, freqüentemente resulta em síndrome de Horner parcial. Os dados de incidência de pacientes com síndrome de Horner parcial neste trabalho são próximos aos obtidos por VAN NES (1986) e KERN et al. (1989) que obtiveram respectivamente $23,1 \%$ e $21,5 \%$. Já GRIFFITHS et al. (1974) e WHEELER et al. (1986) obtiveram respectivamente incidência de $55,6 \%$ e $46,1 \%$.

Apesar da baixa incidência de fraturas simultâneas de rádio ipsilateral e ao fato desta estar mais relacionada à paralisia do nervo radial, ressaltase a importância da pesquisa de sinais que indiquem avulsão de raízes do plexo braquial, como ausência do reflexo do panículo e presença de síndrome de Horner em pacientes apresentados com fraturas de rádio.

As alterações neurológicas mais freqüentes neste estudo e que não são encontradas em cães com paralisia do nervo radial são as seguintes: ausência do reflexo do panículo, atrofia dos músculos bíceps, supra-espinhal e infraespinhal, e anestesia cutânea abaixo da articulação do cotovelo, condizente com as observações de BRAUND (1994).

Segundo GRIFFITHS et al. (1974) e BAILEY (1984) a falta de padronização dos dermátomos em cães dificulta a avaliação das áreas de pele dessensibilizadas. Para SHORES (1993), a sobreposição dos dermátomos, a necessidade de cooperação dos pacientes, e para BAILEY (1984), a variação anatômica e do grau de lesão complicam ainda mais esta situação, concordando com os vários padrões de sensibilidade cutânea observados neste estudo.
Segundo SIMPSON et al. (1985), a alteração sensorial depende da raiz envolvida, sendo que a maior parte dos casos, com avulsão total, apresentaria anestesia distai ao cotovelo. Já BAILEY (1984) observou casos de avulsão total com anestesia distai ao cotovelo e em face cranial de braço, que também foram verificados neste trabalho.

Ressalta-se que através do exame neurológico constatou-se facilmente o acometimento dos nervos radial e músculo cutâneo, através da observação de paralisia flácida, ausência de extensão e flexão de cotovelo, ausência de extensão de carpo e atrofia dos músculos tríceps, bíceps e extensores do carpo. Entretanto, a avaliação da integridade dos nervos medianos e ulnar, depende principalmente da avaliação subjetiva da sensibilidade cutânea. A indicação de técnicas cirúrgicas como panartrodese do carpo e transposição de tendões, nas avulsões parciais, ou seja, com preservação do nervo músculo cutâneo, segundo KNECHT \& RAFFE (1985) teria resultados melhores se a função dos nervos medianos e ulnar fosse determinada previamente.

Apesar dos cuidados dispensados aos pacientes, tais como colocação de talas para evitar lesão à face dorsal da mão, fisioterapia, e uso de colar protetor, a maior parte dos casos apresentou lesões e alterações musculares e articulares graves, havendo a necessidade de indicar a amputação do membro afetado em grande número dos casos, concordando com GRIFFITS (1974), BRAUND (1987), e SHORES (1993). Segundo estes autores, a maioria dos casos apresenta um prognóstico ruim, sendo a progressão da regeneração, se houver, muito lenta, estando de acordo com esta pesquisa, onde o acompanhamento dos casos clínicos demonstrou evolução lenta ou inexistente.

O acompanhamento dos pacientes submetidos a artrodese do carpo demonstrou resultados insatisfatórios, pois apesar da capacidade de flexão do cotovelo estar intacta, não houve por parte dos animais a integração motora e sensorial necessária para apoiar corretamente o membro no solo, decorrente da lesão dos nervos radial, mediano e ulnar. A lesão destes dois últimos nervos favoreceu ainda a ocorrência de ulcerações, concordando com KNECHT \& RAFFE (1985), que citam como principais complicações a atrofia muscular neurogênica, erosão da pele, contratura dos tendões flexores e automutilação.

Os resultados obtidos neste trabalho, associados aos aspectos da histologia e eletroneuroestimulação dos nervos radial, mediano, ulnar e músculo cutâneo, sugeriram envolvimento quase que total das raízes do plexo braquial, indicando a necessidade 
de pesquisas visando descobrir tratamentos eficazes para a recuperação cirúrgica das raízes nervosas.

\section{REFERÊNCIAS BIBLIOGRÁFICAS}

ARCHIBALD, J. Canine surgery. 2 ed. Santa Barbara: American Veterinary Publications, 1974, 1172 p.

BAILEY, C.S. Patterns of cutaneous anesthesia associated with brachial plexus avulsions in the dog. Journal of the American Veterinary Medical Association, v. 185, n. 8, p. 889-899, 1984

BRAUND, K.G. Clinical syndromes in veterinary neurology. Baltimore: Willians \& Wilkins ,1986. 257 p,

BRAUND, K.G. Clinical syndromes in veterinary neurology. St. Louis: Mosby, 1994.477 p.

BRAUND, K.G. Diseases of peripheral nerves, cranial nerves and muscle. In: OLIVER.J.E., HOERLEIN, B.F., MAYHEW, 1.0. Veterinary neurology. Philadelphia: Saunders,1987. p. 353-374.

CARLSTEDT, T.D., HALLIN, R.G., HEDSTROM, K.G. et al. Funcional recovery in primates with brachial plexus injury after spinal cord implantation of avulsed ventral roots. Journal of Neurology, Neurosurgery and Psychiatry, v. 56, p. 649-654, 1993.

CHRISMAN, C.L. Peripheral nerve disorders. In: ETTINGER, S J. Textbook of veterinary internal medicine. Diseases of the dog and cat. Philadelphia: Saunders, 1989, p. 708-732

DE LAHUNTA, A. Veterinary neuroanatomy and clinical neurology. Philadelphia: Saunders, 1983. 471 p.

GRIFFITHS, I.R. Avulsion of the brachial plexus -1. Neuropathology of the spinal cord and peripheral nerves. Journal of Small Animal Practice, v. 15, p. 165-176, 1974

GRIFFITHS, I.A., DUNCAN, I.D., LAWSON, D.D. Avulsion of the brachial plexus - 2. Clinical aspects. Journal of Small Animal Practice, v. 15, p. 177-182, 1974.

GRUMADAS, C.E.S. Pan artrodese do carpo na correção de instabilidade articular provocada experimentalmente por neurectomia do radial em caninos. Santa Maria -RS. 77 p. Dissertação (Mestrado em Cirurgia) - Curso de Pósgraduação em Medicina Veterinária, Universidade Federal de Santa Maria, 1987.

HOERLEIN, B.F., BOWEN, J.M. Clinical disorders of nerves and muscles. In: HOERLEIN, B.F. Canine neurology. 2. ed. Philadelphia: Saunders, 1978, p. 280-295

HOFFMAN, C.F.E., THOMEER, R.T.W.M., MARAN1, E. Ventral root avulsions of the spinal cord at the brachial plexus level (cervical 7). European Journal of Morphology, v. 28, n. $2-4$, p. $418-429,1990$.

KERN, T.J., AROMANDO, M.C., ERB, H.N. Horner's syndrome in dogs and cats: 100 cases (1975-1985). Journal of the American Veterinary Medicai Association, v. 195, n.3, p.369-373, 1989.

KNECHT, C.D., RAFFE, M.R. Diseases of the brachial plexus. In: NEWTON, C.D., NUNAMAKER, D.M. Textbook of small animal orthopedics. Philadelphia: Lippincott, 1985. p. 817-823.

KNECHT, C.D., ST CLAIR, L.E. Brachial paralysis syndrome in the dog. Journal of the American Veterinary Medical Association, v. 154 n. 6 p. 653-656, 1969.

SHORES, A. Traumatic and neoplastic diseases ofthe brachial plexus. In: BOJRAB, M.J. Diseases mechanism in small animais. 2. ed. Philadelphia: Lea\& Febiger, 1993. p. i 1751182 .

SIMPSON, S.T., KORNEGAY, J.N., RAFFE, M.R. Surgical diseases of peripheral nerves. In : SLATTER, D.H. Textbook of small animal surgery. Philadelphia: Saunders, 1985. p. $1335-1338$

STEINBERG, H.S. Brachial plexus injuries and dysfúnctions. Veterinary Clinics of North América. Small Animal Practice, v. 18, n. 3, p. 565-580, 1988.

STOPIGLIA, A.J., ERHART, E.A. Utilização de técnica microcirúrgica na neurorrafia de animais domésticos. Estudo experimental de reparação fascicular no nervo ulnar de cães (Canis familiaris). Revista da Faculdade de Medicina Veterinária e Zootecnia da Universidade de São Paulo, v. 24, n. 2, p. 193-207, 1987.

SUMMERS, B.A., CUMMINGS, J.F., DE LAHUNTA, A Veterinary neuropathology. St Louis: Mosby, 1995. 527 p.

WHEELER, S.J., CLAYTON JONES, D.G., WRIGHT, J.A. The diagnosis of brachial plexus disorders in dogs: a review of twenty-two cases. Journal of Small Animal Practice, v. 27, p.147-157, 1986.

VAN NES, J.J. Electrophysiological evaluation of traumatic forelimb paralysis of the dog. Research in Veterinary Science, v. 40, p. 144-147, 1986. 